\title{
Determinants of progression from impaired fasting glucose and impaired glucose tolerance to diabetes in a high-risk screened population: 3 year follow-up in the ADDITION study, Denmark
}

\author{
S. S. Rasmussen • C. Glümer • A. Sandbaek • \\ T. Lauritzen • K. Borch-Johnsen
}

Received: 17 September 2007 / Accepted: 5 November 2007 / Published online: 5 December 2007

(C) Springer-Verlag 2007

\begin{abstract}
Aims/hypothesis We sought to identify determinants of progression from impaired fasting glucose (IFG) and impaired glucose tolerance (IGT) to diabetes in high-risk screened individuals.

Methods In general practices in Denmark, stepwise screening for type 2 diabetes mellitus in persons aged 40 to 69 years included a risk questionnaire, random blood glucose, $\mathrm{HbA}_{1 \mathrm{c}}$, fasting blood glucose and an OGTT. The 1,821 individuals with IGT or isolated IFG (WHO 1999) were re-invited after 1 and 3 years. Follow-up data on glucose measurements were available in 1,510 individuals and additional clinical data in 1,002 collected at the 3 -year visits. Regression models using interval censoring were used.

Results Progression rates from IFG and IGT to diabetes over 3.5 years were 11.8 and 17.0 per 100 person-years, respectively and were particularly high in the first year. Baseline determinants of progression were: IFG: glucose
\end{abstract}

S. S. Rasmussen $(\bowtie) \cdot K$. Borch-Johnsen

Steno Diabetes Center,

Niels Steensensvej 1,

2820 Gentofte, Denmark

e-mail:SSSR@steno.dk

C. Glümer

Research Center for Prevention and Health,

The Capital Region of Denmark,

Glostrup, Denmark

A. Sandbaek $\cdot$ T. Lauritzen $\cdot$ K. Borch-Johnsen

Institution of Public Health, Department of General Practice,

University of Aarhus,

Aarhus, Denmark measures, BMI $\left[\right.$ per $\mathrm{kg} / \mathrm{m}^{2}$, rate ratio (RR) 1.04 (95\% CI, 1.01-1.08)] and triacylglycerol [per twofold increase, RR 2.19 (1.49-3.22)]; and IGT: glucose measures and known hypertension [RR 1.46 (1.11-1.93)]. Weight reduction and decreased triacylglycerol were inversely associated with development of diabetes in IFG individuals [per $1 \mathrm{~kg} /$ year, RR $0.81(0.66-0.98)$ and per $1 \mathrm{mmol} \mathrm{l}^{-1}$ year $^{-1}$, RR 0.08 (0.01-0.51), respectively], whereas in IGT participants only weight reduction was inversely associated [per $1 \mathrm{~kg} / \mathrm{year}$, RR $0.80(0.67-0.96)]$.

Conclusions/interpretation Higher levels of glucose measures, larger BMI, known hypertension and hypertriacylglycerolaemia are significant determinants of progression in high-risk screened individuals. Weight loss of $1 \mathrm{~kg} /$ year or reduction of hypertriacylglycerolaemia markedly reduced the risk of diabetes.

Keywords Blood glucose · Denmark · Determinants ·

General practice - Impaired fasting glucose .

Impaired glucose tolerance Progression rate .

Screening · Type 2 diabetes mellitus

$\begin{array}{ll}\text { Abbreviations } \\ \text { ADDITION } & \begin{array}{l}\text { Anglo-Danish-Dutch study of Intensive } \\ \text { Treatment in People with Screen Detected } \\ \text { Diabetes in Primary Care } \\ \text { fasting capillary blood glucose }\end{array} \\ \text { FBG } & \begin{array}{l}\text { 2 h capillary blood glucose } \\ \text { 2hBG }\end{array} \\ \text { IFG } & \text { impaired fasting glucose } \\ \text { IGT } & \text { impaired glucose tolerance } \\ \text { RBG } & \text { random capillary blood glucose } \\ \text { RR } & \text { rate ratio }\end{array}$




\section{Introduction}

It is currently being debated whether population screening for impaired glucose tolerance (IGT) and impaired fasting glucose (IFG) is worthwhile in itself. Nevertheless, individuals with IFG or IGT are identified by various screening strategies for type 2 diabetes mellitus. In high-risk screened individuals in general practice we have shown that progression rates to diabetes within the first year are high for both IFG and IGT individuals [1]. Evidence on the risk of developing diabetes in these individuals is essential, as progression to diabetes is preventable with lifestyle changes or pharmacological interventions, at least for persons with IGT [2-9]. By knowing which risk factors or changes in risk factor levels determine progression to diabetes, we may be able to develop individualised focused interventions. Current publications on determinants of progression from IGT have studied population-based cohorts including moderate and low-risk individuals; few studies have reported on IFG [10-16].

We wanted to evaluate possible determinants of progression from isolated IFG and from IGT to diabetes in a highrisk screened Danish population.

\section{Methods}

Study background The Anglo-Danish-Dutch study of Intensive Treatment in People with Screen Detected Diabetes in Primary Care (ADDITION) study is a population-based, high-risk screening and intervention study for type 2 diabetes mellitus in general practice [17]. The screening programme in Denmark and the 1 year follow-up of IFG and IGT have been evaluated previously $[1,18]$. The 1 year follow-up of IFG and IGT included individuals screened from 2001 to August 2003. The present follow-up study includes individuals with IFG and IGT identified in the screening period 2001 to 2005

The screening process The screening process was a stepwise, decision-tree model. Persons aged 40 to 69 years, registered with the participating practices in five counties in Denmark, received an invitation with a risk score questionnaire $[18,19]$. Persons with a risk score of 5 points or more were recommended to contact their general practitioners for further tests. In this second step, random capillary blood glucose (RBG) and $\mathrm{HbA}_{1 \mathrm{c}}$ were measured. Individuals were considered at low risk of having diabetes if RBG $<5.5 \mathrm{mmol} / 1$ and $\mathrm{HbA}_{1 \mathrm{c}}<5.8 \%$. In all those who proceeded in the programme after this second step, fasting capillary blood glucose (FBG) was measured. An OGTT was performed within the same consultation if FBG was 5.6 to $6.1 \mathrm{mmol} / 1$ or $\mathrm{HbA}_{1 \mathrm{c}} \geq 5.8 \%$. Consequently, each step divided persons into high vs low risk of having diabetes. Those at low risk did not continue in the programme. Annual glucose measuring was recommended for individuals with IFG and IGT. Individuals with two diabetic glucose values on separate days were included in the intervention programme.

The follow-up study Invitations for follow-up of glucose measurements in individuals with IFG or IGT were given after 1 and 3 years. After 1 year glucose measures were recorded. At the 3-year visit anthropometric measurements, blood samples and a questionnaire on lifestyle changes were also collected (extended follow-up data). Individuals entering the ADDITION study in 2004 or 2005 were invited to this extended follow-up visit at study-end in 2006. We measured $2 \mathrm{~h}$ capillary blood glucose $(2 \mathrm{hBG})$ regardless of the levels of FBG and $\mathrm{HbA}_{1 \mathrm{c}}$. Individuals with severe concurrent illnesses, alcohol abuse or subsequently being treated by general practitioners not in the ADDITION study were not invited (Fig. 1). Individuals diagnosed with diabetes after screening and before the planned extended follow-up visit were classified as incident cases of diabetes from the day of diagnosis and were not re-invited. During screening, 1,821 individuals were identified with IFG or IGT. Glucose measurements at follow-up were available in 1,510 individuals, for 1,002 of whom extended follow-up data were obtained.

Measurements Three self-administered questionnaires were used: (1) the Danish Risk Score [19]; (2) a baseline questionnaire on medical history, family history of diabetes, smoking status (daily smoker or not) and alcohol consumption; and (3) a follow-up questionnaire on changes since screening. Physical activity was not part of the baseline questionnaire. Baseline physical activity level was therefore based on the risk score questionnaire, in which leisure time activity was considered low when individuals marked 'moderate activity $4 \mathrm{~h}$ a week or less' (physically inactive individuals). Information on medication was not given at baseline. Lifestyle changes since screening were based on recall by the persons, for example in the form of statements on whether their total food intake was much reduced, slightly reduced, unchanged or increased a little or much. These changes were assessed by dichotomised variables indicating any healthy improvement vs no change or worsening. Information on deceased participants was drawn from central registers.

Blood pressure was measured twice with a standard sphygmomanometer after $10 \mathrm{~min}$ rest. The lowest value was recorded. Height and weight were measured with the person wearing light clothing and no shoes. Waist and hip circumferences were measured.

Capillary whole-blood glucose was measured using a glucose dehydrogenase reaction (HemoCue, Ängelholm, Sweden). The average of results from two capillary blood samples taken $1 \mathrm{~min}$ apart was used. The persons were 
Fig. 1 Attendance at the followup visits of individuals with IFG or IGT in the ADDITION study, Denmark. Fasting and 2hBG were measured at all follow-up visits. The 3-year visit (or at study-end for those more recently screened) was extended to include anthropometric measurements, blood samples and a questionnaire with focus on changes in lifestyle since screening. GP stopped: individuals attending general practitioners who stopped participation in the ADDITION study or were unable to do OGTT

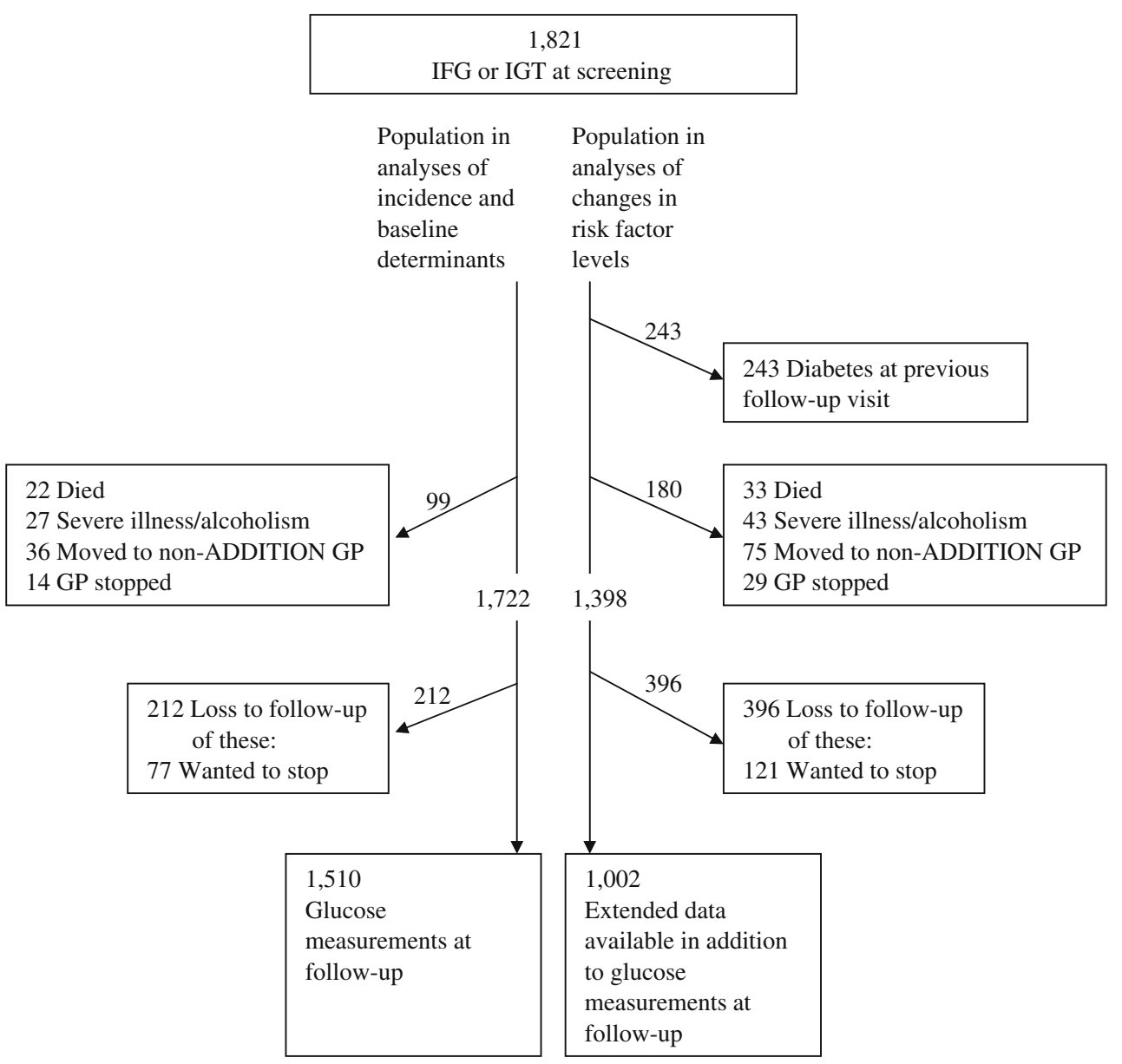

fasting from 23:00 hours on the evening before, having been instructed not to eat, drink or smoke. A 75 g OGTT was performed on the persons staying in the consultation.

Fasting venous blood samples were mailed to the central laboratory (University Hospital of Aarhus). $\mathrm{HbA}_{1 \mathrm{c}}$ was analysed by liquid chromatography (TOSOH $\mathrm{A}_{1 \mathrm{c}} 2.2$; TOSOH/ Eurogenetics, Stuttgart, Germany). The Hitachi 971 system (Roche Diagnostics, Mannheim, Germany) was used to measure total serum cholesterol, serum HDL-cholesterol and serum triacylglycerol by enzymatic test. Serum LDLcholesterol was calculated using Friedewald's formula [20].

Ethical permission The study complied with the Helsinki Declaration and was approved by the local scientific ethics committee in Aarhus (no. 20000183). All participants gave written informed consent.

Definitions Glucose tolerance was classified by the WHO (1999) definition using fasting (FBG) and $2 \mathrm{~h}$ capillary whole blood glucose (2hBG) [21]. Hence, the definition of IFG corresponds to that of isolated IFG $(5.6 \mathrm{mmol} / 1 \leq \mathrm{FBG}$ $<6.1 \mathrm{mmol} / 1$ and $2 \mathrm{hBG}<7.8 \mathrm{mmol} / \mathrm{l}$ ), whereas IGT included isolated IGT and combined IFG and IGT $(\mathrm{FBG}<$
$6.1 \mathrm{mmol} / 1$ and $7.8 \mathrm{mmol} / 1 \leq 2 \mathrm{hBG}<11.1 \mathrm{mmol} / \mathrm{l})$. Incident diabetes was defined as one diabetic value of FBG ( $\geq 6.1 \mathrm{mmol} / \mathrm{l})$ or $2 \mathrm{hBG}(\geq 11.1 \mathrm{mmol} / \mathrm{l})$.

Statistical analyses Differences in risk factor levels between screening and last follow-up are given as follow-up value minus screening value. Continuous variables within groups were compared with two-sided paired $t$ test and categorical variables with $\chi^{2}$ test. Cumulative risks, progression rates and rate ratios (RR) of progression to diabetes were estimated with a regression model using interval censoring [22, 23]; this model is implemented in the EPI package [24] in $\mathrm{R}$ [25]. An approximation of person-years used in the model is: person-years (py) $=$ (last known non-diabetes date screening date $)+($ conversion date - last known non-diabetes date)/2. Analyses were performed for IFG and IGT separately.

Incidence model Progression rates were estimated in intervals of observation years as follows: 0 to $1.5 ;<1.5$; and the total observation period. These intervals include the delay between receipt of an invitation to a general practitioner consultation and the actual appointment. Most visits were made within 2 months. 
Baseline determinant model Screening variables (age, sex, BMI, known hypertension, family history of diabetes, physical inactivity, $\mathrm{RBG}$ and $\mathrm{HbA}_{1 \mathrm{c}}$ ) were included in the model. The model was therefore based on prognostic factors from the screening steps upon which the individuals were selected. Gestational diabetes was not included due to too few exposed individuals. Additional baseline determinants added separately in the model were: FBG, $2 \mathrm{hBG}$, waist circumference, WHR, systolic and diastolic blood pressure, total cholesterol, HDL- and LDL-cholesterol, $\log _{\mathrm{e}}$ (triacylglycerol), smoking and previous myocardial infarction. These separate analyses of additional variables were thus adjusted for the prognostic factors from the screening steps. Additional variables with $p \leq 0.20$ were included in a stepwise backward elimination procedure, where the screening variables were not eliminated. A value of $p<0.05$ was considered significant in the final model.

Time-dependent change-determinant models Effect of changes between screening and follow-up was tested with the difference of a covariate divided by the time from screening to follow-up. Each change covariate was analysed separately adjusted for age, sex and baseline value of the covariate. The covariates tested were: (1) decreased: weight, systolic blood pressure, total cholesterol, LDL-cholesterol, triacylglycerol, smoking, physical inactivity, total food intake and intake of sugar, fat, meat and alcohol; (2) increased: HDLcholesterol, intake of vegetables, fruits and fish, and addition of blood pressure- and cholesterol-lowering medication.

\section{Results}

Of the persons eligible for follow-up, $88 \%(1,510 / 1,722)$ attended at least one follow-up visit (Fig. 1). The 3-year visit, or the end of study visit if not followed for 3 years, was conducted as an extended visit and $72 \%(1,002 / 1,398)$ of those eligible for these visits attended. Of the attendees at follow-up, $25 \%$ were daily smokers and $80 \%$ had stated low physical activity at screening, compared with $37 \%$ ( $p<$ $0.001)$ and $89 \%(p<000.1)$, respectively, for non-attendees. No significant difference in screening values for age, sex, BMI, systolic blood pressure and total cholesterol was seen between attendees at follow-up and non-attendees.

There were 442 incident diabetes cases. Progression rates to diabetes were 11.8 and 17.0 per 100 person-years for IFG and IGT, respectively (Table 1), which corresponds to cumulative risks of 32 and $41 \%$ (Fig. 2). Progression rates were remarkably higher in the first year than in the rest of the observation period. Separating IGT into isolated IGT and combined IFG and IGT, we found the following progression rates for isolated IFG, isolated IGT and combined IFG and IGT, respectively: observation years $0-1.5$ : 16.0 (95\% CI, 13.1-19.0), 15.7 (12.6-18.9) and 38.2 (31.4$45.1)$; observation years $<1.5$ years: 6.1 (4.0-8.2), $6.3(4.0$ 8.6) and 9.6 (5.4-13.9); and total period: 11.8 (9.9-13.8), 11.8 (9.7-13.9) and 27 (22.5-31.7) per 100 person-years.

In the baseline determinant model with eight screening variables, the following significant associations were found between incident diabetes and IFG: BMI (per $\mathrm{kg} / \mathrm{m}^{2}: \mathrm{RR}=$ $1.05, p=0.005$ ), physical inactivity (yes vs no: $\mathrm{RR}=1.68$, $p=0.025$ ) and $\mathrm{HbA}_{1 \mathrm{c}}$ (per 0.5\%: $\mathrm{RR}=1.59, p<0.001$ ). Significant associations between incident diabetes and IGT were: BMI (per $\mathrm{kg} / \mathrm{m}^{2}$ : $\mathrm{RR}=1.03, p=0.022$ ), known hypertension (yes vs no: $\mathrm{RR}=1.33, p=0.040$ ), $\mathrm{RBG}$ (per $0.5 \mathrm{mmol} / \mathrm{l}: \mathrm{RR}=1.09, p<0.001$ ) and $\mathrm{HbA}_{1 \mathrm{c}}$ (per $0.5 \%$ : $\mathrm{RR}=$ $1.36, p<0.001)$. When including $2 \mathrm{hBG}$ in the IFG model and FBG in the IGT model, effects of physical inactivity and both BMI and RBG, respectively, became statistically insignificant (Table 2). Separating IGT into isolated IGT and combined IFG and IGT, respectively, affected the estimates from FBG, $2 \mathrm{hBG}$ and triacylglycerol. From the separate analyses adjusted for screening variables, these
Table 1 Baseline characteristics and progression rates from IFG and IGT (WHO 1999 [21]) to diabetes

Unless otherwise indicated, data are means \pm SD or percentage $(95 \% \mathrm{CI})$

${ }^{\mathrm{a}}$ Geometric mean (coefficient of variation)

${ }^{\mathrm{b}}$ Median (interquartile range); person-years $=$ (last known non-diabetes date - screening date $)+($ conversion date - last known non-diabetes date)/2

\begin{tabular}{|c|c|c|}
\hline & IFG & IGT \\
\hline \multicolumn{3}{|l|}{ Baseline characteristics } \\
\hline$N$ & 607 & 903 \\
\hline Sex, \% women $(n)$ & $43(260)$ & $56(504)$ \\
\hline Age (years) & $59.9 \pm 6.3$ & $61.2 \pm 6.6$ \\
\hline BMI $\left(\mathrm{kg} / \mathrm{m}^{2}\right)$ & $29.1 \pm 4.7$ & $29.6 \pm 5.0$ \\
\hline Systolic blood pressure $(\mathrm{mmHg})$ & $140 \pm 18$ & $142 \pm 18$ \\
\hline Total cholesterol $(\mathrm{mmol} / \mathrm{l})$ & $5.7 \pm 1.0$ & $5.9 \pm 1.1$ \\
\hline Triacylglycerol $(\mathrm{mmol} / \mathrm{l})^{\mathrm{a}}$ & $1.2(0.5)$ & $1.4(0.5)$ \\
\hline \multicolumn{3}{|c|}{ Progression rates (cases per 100 person-years) } \\
\hline Observation period: $0-1.5$ years & $16.0(13.1-19.0)$ & $23.8(20.7-26.9)$ \\
\hline Observation period: $>1.5$ years & $6.1(4.0-8.2)$ & $7.2(5.2-9.3)$ \\
\hline Total observation period & $11.8(9.9-13.8)$ & $17.0(14.9-19.1)$ \\
\hline Median follow-up time (person-years) ${ }^{b}$ & $2.5(1.3-3.0)$ & $2.1(0.7-2.9)$ \\
\hline
\end{tabular}




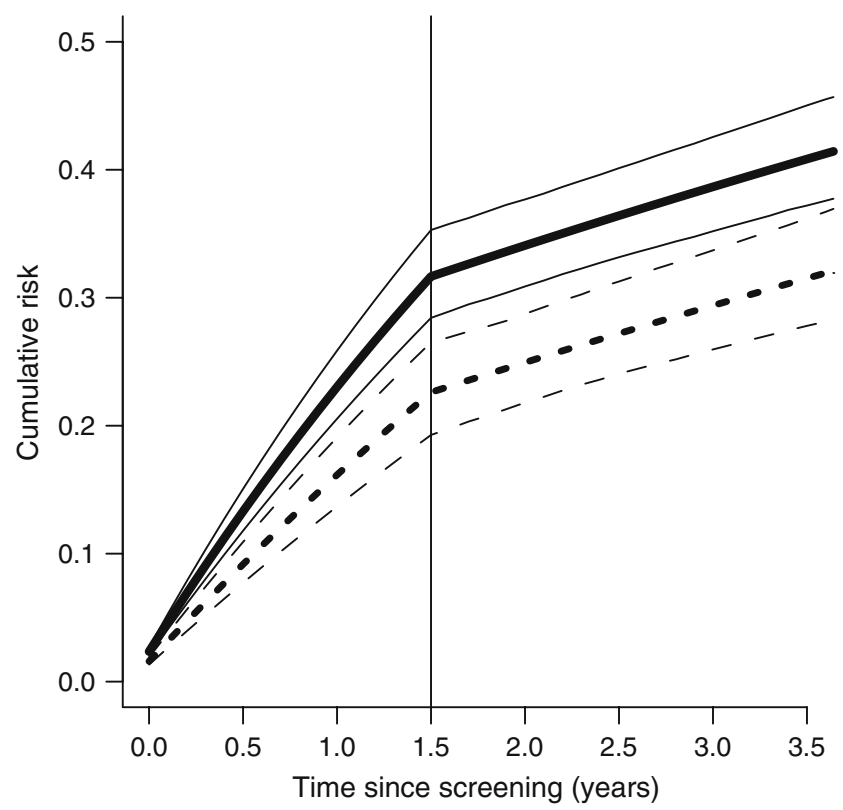

Fig. 2 Estimated cumulative risk of progression from IFG or IGT to diabetes in the high-risk screened population of the ADDITION study. The curves are based on regression analyses using interval censoring. Continuous lines, IGT and 95\% CIs; dashed lines, IFG and 95\% CIs. Vertical line represents first-year visit

estimates were: FBG RR 1.92 (95\% CI, 1.16-3.19) and 3.78 (2.17-6.58), 2hBG $1.40(1.27-1.54)$ and $1.16(1.06-1.27)$, and triacylglycerol $1.69(1.07-2.65)$ and $0.94(0.61-1.46)$ for isolated IGT and combined IFG and IGT, respectively.
Table 3 shows changes in risk factor levels and estimated $\mathrm{RR}$ of progression from IFG and IGT to diabetes. The analyses presented were made on the complete dataset for all variables tested $(n=498)$. The number of observations was considerably reduced. The same analyses were therefore conducted on separate datasets including only the variables in the respective analyses $(n \geq 823)$ (not shown). Analyses on the complete dataset reached statistical significance more frequently. Point estimates for triacylglycerol and HDL-cholesterol were reduced in the analyses performed on separate datasets, whereas the confidence intervals in the two strategies overlapped, indicating that there was no statistical difference between the estimates.

\section{Discussion}

This high-risk screened population with isolated IFG and IGT identified in general practice had a cumulative risk of 32 and $41 \%$ respectively of developing diabetes within 3.5 years. Progression rates were particularly high in the first year (about 20 per 100 person-years) and remained high in both groups. Higher levels of any glucose measures were seen to independently determine progression. Additional determinants of progression in the isolated IFG group were BMI and triacylglycerol. In the IGT group, known hypertension increased the risk by almost $50 \%$ compared with participants without known hypertension. In compari-
Table 2 Baseline determinants of progression from IFG and IGT (WHO, 1999 [21]) to diabetes

BG Blood glucose

${ }^{\text {a }}$ Separate analyses of additional variables adjusted for screening variables

${ }^{\mathrm{b}}$ Final model after backward elimination procedure for additional variables, where those significant at $p<0.05$ are shown ${ }^{c}$ Triacylglycerol was not significantly associated with progression in the IGT group in the final model

${ }^{\mathrm{d}} p<0.05$

e $p<0.01$

${ }_{p}^{\mathrm{f}}<0.001$

\begin{tabular}{|c|c|c|}
\hline Risk factor by variables & IFG RR $(95 \%$ CI $)$ & IGT RR $(95 \% \mathrm{CI})$ \\
\hline \multicolumn{3}{|l|}{ Separate analyses of additional variables ${ }^{\mathrm{a}}$} \\
\hline Fasting BG (per $0.5 \mathrm{mmol} / \mathrm{l})$ & $3.45(2.58-4.63)^{\mathrm{e}}$ & $1.67(1.45-1.92)^{\mathrm{e}}$ \\
\hline $2 \mathrm{hBG}($ per $0.5 \mathrm{mmol} / \mathrm{l})$ & $1.13(1.03-1.23)^{\mathrm{d}}$ & $1.27(1.19-1.36)^{\mathrm{e}}$ \\
\hline Waist (per cm) & $1.02(0.99-1.05)$ & $1.00(0.98-1.02)$ \\
\hline Systolic blood pressure (per mmHg) & $1.01(1.00-1.02)^{\mathrm{d}}$ & $1.00(0.99-1.00)$ \\
\hline Diastolic blood pressure (per mmHg) & $1.02(1.00-1.03)$ & $1.00(0.99-1.01)$ \\
\hline Total cholesterol (per mmol/l) & $1.33(1.10-1.61)^{\mathrm{d}}$ & $0.93(0.82-1.05)$ \\
\hline Triacylglycerol (per twofold increase) & $2.10(1.44-3-06)^{\mathrm{e}}$ & $1.17(0.86-1.60)$ \\
\hline History of myocardial infarction (yes vs no) & $1.36(0.41-4.54)$ & $1.33(0.79-2.24)$ \\
\hline Daily smoking (yes vs no) & $1.52(1.03-2.25)^{\mathrm{d}}$ & $1.15(0.84-1.59)$ \\
\hline \multicolumn{3}{|l|}{ Final model: screening variables ${ }^{\mathrm{b}}$} \\
\hline Sex (men vs women) & $1.03(0.6-1.52)$ & $0.79(0.60-1.04)$ \\
\hline Age (per 5 years) & $1.08(0.93-1.26)$ & $0.99(0.89-1.09)$ \\
\hline BMI (per kg/m²) & $1.04(1.01-1.08)^{\mathrm{d}}$ & $1.02(0.99-1.05)$ \\
\hline Known hypertension (yes vs no) & $1.02(0.70-1.49)$ & $1.46(1.11-1.93)^{\mathrm{e}}$ \\
\hline Family history of diabetes (yes vs no) & $1.15(0.78-1.70)$ & $1.17(0.88-1.55)$ \\
\hline Physical inactivity (yes vs no) & $1.54(0.97-2.47)$ & $1.14(0.79-1.64)$ \\
\hline Random BG (per $0.5 \mathrm{mmol} / \mathrm{l})$ & $0.98(0.90-1.07)$ & $1.04(0.99-1.10)$ \\
\hline $\mathrm{HbA}_{1 \mathrm{c}}($ per $0.5 \%)$ & $1.40(1.09-1.80)^{\mathrm{e}}$ & $1.23(1.08-1.47)^{\mathrm{e}}$ \\
\hline \multicolumn{3}{|l|}{ Final model: additional variables ${ }^{\mathrm{b}}$} \\
\hline Fasting BG (per $0.5 \mathrm{mmol} / \mathrm{l})$ & $3.19(2.33-4.37)^{\mathrm{f}}$ & $1.65(1.43-1.92)^{\mathrm{f}}$ \\
\hline $2 \mathrm{hBG}($ per $0.5 \mathrm{mmol} / \mathrm{l})$ & $1.10(1.00-1.21)^{\mathrm{d}}$ & $1.26(1.18-1.35)^{\mathrm{f}}$ \\
\hline Triacylglycerol (per twofold increase) ${ }^{\mathrm{c}}$ & $2.19(1.49-3.22)^{\mathrm{f}}$ & - \\
\hline
\end{tabular}


Table 3 Time-dependent changes in risk factors as determinants of progression from IFG and IGT (WHO 1999 [21]) to diabetes in separate analyses

\begin{tabular}{|c|c|c|c|c|}
\hline \multirow[t]{2}{*}{ Risk factor } & \multicolumn{2}{|l|}{ IFG } & \multicolumn{2}{|l|}{ IGT } \\
\hline & $\begin{array}{l}\text { Change from } \\
\text { screening }\end{array}$ & $\mathrm{RR}(95 \% \mathrm{CI})$ & $\begin{array}{l}\text { Change from } \\
\text { screening }\end{array}$ & $\mathrm{RR}(95 \% \mathrm{CI})$ \\
\hline Weight (RR: per decreased kg/year) ${ }^{\mathrm{a}}$ & $0.10(-0.41,0.60)$ & $0.81(0.66,0.98)^{\mathrm{c}}$ & $-0.84(-1.23,-0.46)^{\mathrm{e}}$ & $0.80(0.67,0.96)^{\mathrm{c}}$ \\
\hline Systolic BP (RR: per decreased $5 \mathrm{mmHg} /$ year) ${ }^{\mathrm{a}}$ & $-3.7(-5.3,-2.1)^{\mathrm{e}}$ & $1.25(0.91,1.71)$ & $-3.3(-4.8,-1.71)^{\mathrm{e}}$ & $0.92(0.72,1.16)$ \\
\hline $\begin{array}{l}\text { Total cholesterol (RR: per decreased } \\
\left.\mathrm{mmol} \mathrm{l}^{-1} \text { year }^{-1}\right)^{\mathrm{a}}\end{array}$ & $-0.09(-0.17,-0.00)^{\mathrm{c}}$ & $1.33(0.46,3.85)$ & $-0.24(-0.33,-0.15)^{\mathrm{e}}$ & $3.80(1.65,8.74)^{\mathrm{d}}$ \\
\hline $\begin{array}{l}\text { HDL-cholesterol (RR: per increased } \\
\left.\mathrm{mmol} \mathrm{l}^{-1} \text { year }^{-1}\right)^{\mathrm{a}}\end{array}$ & $0.02(-0.00,0.05)$ & $0.74(0.01,37.18)$ & $0.07(0.04,0.09)^{\mathrm{e}}$ & $0.02(0.00,0.80)^{\mathrm{c}}$ \\
\hline $\begin{array}{l}\text { LDL-cholesterol (RR: per decreased } \\
\left.\mathrm{mmol} \mathrm{l}^{-1} \text { year }^{-1}\right)^{\mathrm{a}}\end{array}$ & $-0.13(-0.2,-0.06)^{\mathrm{e}}$ & $2.11(0.86,7.18)$ & $-0.29(-0.37,-0.21)^{\mathrm{e}}$ & $3.72(1.34,10.31)^{\mathrm{c}}$ \\
\hline 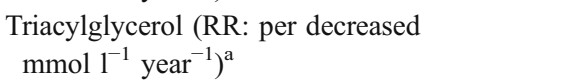 & $0.07(-0.01,0.15)$ & $0.08(0.01,0.51)^{\mathrm{d}}$ & $-0.05(-0.11,-0.00)^{\mathrm{c}}$ & $1.12(0.27,4.60)$ \\
\hline Added BP medication (RR: yes vs no) ${ }^{\mathrm{b}}$ & $16.6(13.0,20.8)$ & $1.69(0.69,4.12)$ & $20.6(17.1,24.6)$ & $1.68(0.85,3.33)$ \\
\hline Added cholesterol medication (RR: yes vs no) ${ }^{b}$ & $9.7(6.9,13.1)$ & $2.13(0.74,6.10)$ & $19.0(15.6,22.8)$ & $2.79(1.44,5.40)^{\mathrm{d}}$ \\
\hline More physical active (RR: yes vs no) ${ }^{\mathrm{b}}$ & $24.4(20.4,28.9)$ & $1.63(0.83,3.21)$ & $28.9(25.1,33.0)$ & $1.09(0.60,1.97)$ \\
\hline Less total food intake (RR: yes vs no) ${ }^{\mathrm{b}}$ & $22.0(18.1,26.2)$ & $1.11(0.51,2.44)$ & $34.9(30.9,39.1)$ & $1.21(0.68,2.14)$ \\
\hline Less smoking (RR: yes vs no) ${ }^{\mathrm{b}}$ & $10.3(7.3,13.9)$ & $0.54(0.18,1.16)$ & $9.8(7.3,12.9)$ & $2.60(1.00,6.76)$ \\
\hline
\end{tabular}

Due to missing data, $n=498$. Rate ratios (RR) are adjusted for age, sex and baseline value of the covariate (except for total food intake). The following age and sex-adjusted RRs in other variables tested were statistically insignificant: intake of sugar, fat, meat, alcohol, vegetables, fruits and fish

${ }^{\mathrm{a}}$ Mean $(95 \% \mathrm{CI})$

${ }^{\mathrm{b}}$ Percentage $(95 \% \mathrm{CI})$

${ }^{\mathrm{c}} p<0.05$

$\mathrm{d}_{p}<0.01$

${ }^{\mathrm{e}} p<0.001$

son with stable weight and triacylglycerol, weight reduction of $1 \mathrm{~kg} /$ year was associated, independently of baseline weight, with about $20 \%$ reduced risk in both groups, while a decrease in triacylglycerol reduced the risk of diabetes in the IFG group. The effect of changes in lipid levels in persons with IGT was equivocal, as healthy changes in LDLand HDL-cholesterol had opposite effects on the risk of diabetes. In individuals who started on cholesterol-lowering medication the risk of diabetes more than doubled (in IFG statistically insignificantly).

Attendees had a healthier lifestyle than non-attendees. This may lead to an underestimation of the progression rates. Nevertheless, attendees and non-attendees did not differ concerning other major risk factors tested. Follow-up rates were comparable with those in other studies of followup [26-28]. Questionnaires were self-administered with risk of recall bias. We believe that this is a minor concern for interpretation of the results, as self-reported lifestyle changes corresponded to objective measurement results in our study. Analyses on time-dependent changes were done on a complete but reduced dataset due to missing values. This affected the size of the point estimates of changes in HDL-cholesterol and triacylglycerol, although not statistically significantly. For this reason, interpretation of the strength of these two estimated RRs must be conservative.
We estimated progression rates with a regression analysis using interval censoring $[22,23]$, which within small time intervals calculates the possibility of a person developing diabetes. As far as we are aware, interval censoring or how person-years were calculated is described in only few publications on progression rates to diabetes $[16,29]$. This may indicate that the remaining studies $[16,26,28,30,31]$ underestimate the true progression rates because of conservatively calculated risk times. We evaluated incident diabetes epidemiologically by one FBG or $2 \mathrm{hBG}$. Previously we have shown that the diagnosis was confirmed in $62 \%$ of the cases [1].

The main reason for the high progression rates found in our study must be that the population was identified by a high-risk approach using a questionnaire on diabetes status. Prevention trials in IGT were also based on high-risk individuals, with progression rates in the control groups also higher than those in population-based studies [2-5]. The decline in progression rates after the first year may reflect the possibility that many of the individuals were close to the diabetic threshold at the time of screening.

In our study, categorisation was based on the WHO (1999) definition [20]. Both $\mathrm{FBG}$ and $2 \mathrm{hBG}$ were required. In accordance with clinical practice and national guidelines, the definition of IFG was based on isolated FBG elevation 
and IGT on $2 \mathrm{hBG}$ regardless of FBG. When categorisations are based on FBG alone, the $2 \mathrm{hBG}$ value is elevated in an unknown proportion of individuals with IFG. Because the definition or method of diagnosis used vary between countries, we also separated the IGT group into isolated IGT and combined IFG and IGT. Progression rates for isolated IGT were similar to those for isolated IFG and progression rates for the combined IFG and IGT group were higher in each observation period.

Fewer determinants of progression were identified in the IGT group than in population-based studies, where most consistently the following were identified: higher levels of blood glucose $\left(\mathrm{HbA}_{1 \mathrm{c}}, \mathrm{FBG}, 2 \mathrm{hBG}\right)$, obesity (BMI and WHR), known hypertension and ethnicity [12, 13, 15]. However, the Hoorn study only found $2 \mathrm{hBG}$ and fasting proinsulin to be predictors of progression [14]. After adding FBG to the model, the association with BMI in our study became insignificant.

Our study is the first to report on determinants of progression from isolated IFG to diabetes. Higher levels of glucose measures, BMI and triacylglycerol were associated with progression in the final model. These determinants were also among those found in a study of progression from incident IFG, though not excluding IGT [16].

Determinants of progression to diabetes may differ between glucose intolerance categories as the physiology of isolated IFG differs from that of isolated IGT [32]. The baseline determinant analyses for the two groups of isolated IGT and combined IFG and IGT emphasised the effects of glucose measures in the risk of developing diabetes. In parallel with the analyses in IFG, increasing levels of triacylglycerol increased the risk of diabetes in the isolated IGT group, but not in the combined IFG and IGT group.

We assessed physical activity by the risk questionnaire, classifying individuals crudely as having high or low physical activity. The majority of participants was physically inactive and physical inactivity was not associated with progression from IGT (or from isolated IGT). More extensive questionnaires have shown an inverse association between physical activity and risk of progressing from IGT to diabetes[33]. The effect of physical inactivity became insignificant in the IFG group after adding $2 \mathrm{hBG}$ to the model, which may reflect the possibility that physical activity and $2 \mathrm{hBG}$ are associated due to physiological mechanisms in peripheral insulin sensitivity [34].

Hence, in this high-risk screened population prediction of progression from IFG or IGT to diabetes could be based on few baseline determinants. It appears that the more rapid the progression to diabetes, the fewer the risk factors that contribute to determining this progression. For example, the combined IFG and IGT group had the highest progression rate, yet the fewest determinants for progression were identified in this group. These individuals were at such high risk that the metabolic state must have been close to the diabetic threshold.

The high progression rates within a short time in our high-risk population may not have left time for changes in risk factor levels to affect the development of diabetes. Notwithstanding this, we showed that in individuals with IFG or IGT, even small weight reductions reduced the risk of developing diabetes compared with no reduction (stable weight). However, a short-term study, not stating baseline glucose tolerance, did not find a beneficial effect of weight reduction [35], whereas a lifestyle intervention study in IGT supports our findings, as risk reduction was $16 \%$ per $\mathrm{kg}$ of reduced weight [36]. Furthermore, weight change as a continuous covariate increased the risk of diabetes from maximum weight loss to maximum weight gain in a study with unknown baseline glucose tolerance status [37].

Based on the effect of self-report of added medication and change in cholesterol levels, the need for cholesterolmodifying medication seemed to increase the risk of diabetes compared with being untreated, this being particularly so in individuals with IGT. These effects may be interpreted as effects of confounding by indication. Individuals receiving treatment and improving the lipid profile may in a way have a worse overall risk profile. We therefore do not conclude that treating dyslipidaemia was a risk factor. By contrast, i.e. in the IFG group, we found that decreasing levels of triacylglycerol reduced the risk of diabetes. Lifestyle changes are associated with changes in triacylglycerol [38]. Yet, decreasing triacylglycerol independently reduced the risk of diabetes in our study, when adjusted for change in alcohol consumption or weight change (not shown). Another observational study has shown that decreases in triacylglycerol decreased the risk of coronary heart disease, independently of several lifestyle changes and medication, when compared with stable high triacylglycerol [38]. Based on our results, future studies may look further into the degree to which changes in triacylglycerol determine progression to diabetes, as we were unable to sufficiently adjust for lifestyle changes.

Based on the high cumulative risk of diabetes within 3.5 years in this study in very high-risk individuals, it could be argued that prediction of progression to diabetes is meaningless. The decline in progression rates after the first year indicates that some individuals progress more rapidly than others and therefore prediction of rapid vs slow progression from IFG and IGT to diabetes would make more sense in this setting, with regard to risk assessment and prevention. We look forward to evaluating this issue in a future paper.

In conclusion, individuals with IFG or IGT identified through high-risk strategies have a very high risk of developing diabetes within the first 3.5 years and this is most pronounced in first year of follow-up. We found that in addition to higher levels of glucose measures special attention 
is indicated by the presence of larger BMI, known hypertension and hypertriacylglycerolaemia. These risk factors can be readily assessed by general practitioners. Risk assessment and preventive initiatives can and should thus be performed at the time of identification. As weight loss of $1 \mathrm{~kg}$ per year or reduction of hypertriacylglycerolaemia markedly reduced the risk of diabetes compared with stable conditions, diabetes is potentially preventable in white, high-risk screened individuals with IFG or IGT in general practice.

Acknowledgements We thank B. Carstensen for his advice on the statistical analyses, and all general practitioners and their staff for making this study possible. This fully investigator-initiated, designed and controlled study received funding from: the Danish Center for Evaluation and Health Technology Assessment; the Danish Research Foundation for General Practice; the counties of Aarhus, Copenhagen, Ringkoebing, Ribe and South Jutland; the National Board of Health; the Danish Medical Research Council no 22-04-0390; the Danish Diabetes Association; the A.P. Møller Foundation; Novo Nordisk A/S and Novo Nordisk Scandinavia AB; Astra-Zeneca; Pfizer; Servier; GlaxoSmithKline; and HemoCue.

Duality of interest K. Borch-Johnsen is employed by and holds shares in Novo Nordisk A/S. He has received lecture fees from Novo Nordisk, Bristol-Myers Squibb, Novartis, Pfizer, Hermedico and AstraZeneca. T. Lauritzen has received lecture fees of approximately $€ 5,000$ during the past year from Novo Nordisk A/S; he holds no shares in or has done consultancy work for Novo Nordisk AS or any other medical industry. C. Glümer holds shares in Novo Nordisk A/S. The authors declare that there is no other duality of interest associated with this manuscript.

\section{References}

1. Rasmussen SS, Glümer C, Sandbaek A, Lauritzen T, BorchJohnsen K (2007) Progression from impaired fasting glucose and impaired glucose tolerance to diabetes in a high-risk screening programme in general practice. The ADDITION study, Denmark. Diabetologia 50:293-297

2. Tuomilehto J, Lindstrom J, Eriksson JG et al (2001) Prevention of type 2 diabetes mellitus by changes in lifestyle among subjects with impaired glucose tolerance. N Engl J Med 344:343-1350

3. Knowler WC, Barrett-Connor E, Fowler SE et al (2002) Reduction in the incidence of type 2 diabetes with lifestyle intervention or metformin. N Engl J Med 346:393-403

4. Chiasson JL, Josse RG, Gomis R et al (2002) Acarbose for prevention of type 2 diabetes mellitus: the STOP-NIDDM randomised trial. Lancet 359:2072-2077

5. Pan XR, Li GW, Hu YH et al (1997) Effects of diet and exercise in preventing NIDDM in people with impaired glucose tolerance. The Da Qing IGT and Diabetes Study. Diabetes Care 20:537-544

6. Kosaka K, Noda M, Kuzuya T (2005) Prevention of type 2 diabetes by lifestyle intervention: a Japanese trial in IGT males. Diabetes Res Clin Pract 67:152-162

7. The Diabetes Prevention Program Research Group (2005) Prevention of type 2 diabetes with troglitazone in the Diabetes Prevention Program. Diabetes 54:1150-1156

8. Ramachandran A, Snehalatha C, Mary S et al (2006) The Indian Diabetes Prevention Programme shows that lifestyle modification and metformin prevent type 2 diabetes in Asian Indian subjects with impaired glucose tolerance (IDPP-1). Diabetologia 49:289297

9. Gerstein HC, Yusuf S, Bosch J et al (2006) Effect of rosiglitazone on the frequency of diabetes in patients with impaired glucose tolerance or impaired fasting glucose: a randomised controlled trial. Lancet 368:1096-1105

10. Bengtsson C, Blohme G, Lapidus L, Lissner L, Lundgren H (1992) Diabetes incidence in users and non-users of antihypertensive drugs in relation to serum insulin, glucose tolerance and degree of adiposity: a 12-year prospective population study of women in Gothenburg, Sweden. J Intern Med 231:583-588

11. Chen KT, Chen CJ, Gregg EW, Imperatore G, Narayan KM (2003) Impaired fasting glucose and risk of diabetes in Taiwan: follow-up over 3 years. Diabetes Res Clin Pract 60:177-182

12. Edelstein SL, Knowler WC, Bain RP et al (1997) Predictors of progression from impaired glucose tolerance to NIDDM: an analysis of six prospective studies. Diabetes 46:701-710

13. Little RR, England JD, Wiedmeyer HM et al (1994) Glycated haemoglobin predicts progression to diabetes mellitus in Pima Indians with impaired glucose tolerance. Diabetologia 37:252-256

14. Nijpels G, Popp-Snijders C, Kostense PJ, Bouter LM, Heine RJ (1996) Fasting proinsulin and 2-h post-load glucose levels predict the conversion to NIDDM in subjects with impaired glucose tolerance: the Hoorn Study. Diabetologia 39:113-118

15. Rajala U, Qiao Q, Laakso M, Keinanen-Kiukaanniemi S (2000) Antihypertensive drugs as predictors of type 2 diabetes among subjects with impaired glucose tolerance. Diabetes Res Clin Pract 50:231-239

16. Nichols GA, Hillier TA, Brown JB (2007) Progression from newly acquired impaired fasting glucose to type 2 diabetes. Diabetes Care 30:228-233

17. Lauritzen T, Griffin S, Borch-Johnsen K et al (2000) The ADDITION study: proposed trial of the cost-effectiveness of an intensive multifactorial intervention on morbidity and mortality among people with Type 2 diabetes detected by screening. Int J Obes Relat Metab Disord 24(Suppl 3):S6-S11

18. Christensen JO, Sandbaek A, Lauritzen T, Borch-Johnsen K (2004) Population-based stepwise screening for unrecognised Type 2 diabetes is ineffective in general practice despite reliable algorithms. Diabetologia 47:1566-1573

19. Glumer C, Carstensen B, Sandbaek A, Lauritzen T, Jorgensen T, Borch-Johnsen K (2004) A Danish diabetes risk score for targeted screening: the Inter99 study. Diabetes Care 27:727-733

20. Friedewald WT, Levy RI, Fredrickson DS (1972) Estimation of the concentration of low-density lipoprotein cholesterol in plasma, without use of the preparative ultracentrifuge. Clin Chem 18:499502

21. WHO (1999) Definition, diagnosis and classification of diabetes mellitus and its complications. Report of a WHO Consultation, Part 1: Diagnosis and classification of diabetes mellitus. WHO, Geneva

22. Carstensen B (1996) Regression models for interval censored survival data: application to HIV infection in Danish homosexual men. Stat Med 15:2177-2189

23. Farrington CP (1996) Interval censored survival data: a generalized linear modelling approach. Stat Med 15:283-292

24. Carstensen B, Plummer M, Läärä E et al (2007) EPI: A package for statistical analysis in epidemiology. R package version 0.7.3. http://www.pubhealth.ku.dk/ bxc/Epi/. Cited 11 September 2007

25. R Development Core Team (2006) R: a language and environment for statistical computing. R Foundation for Statistical Computing, Vienna. http://www.R-project.org. Cited 11 September 2007

26. de Vegt F, Dekker JM, Jager A et al (2001) Relation of impaired fasting and postload glucose with incident type 2 diabetes in a Dutch population: The Hoorn Study. JAMA 285:2109-2113 
27. Forouhi NG, Luan J, Hennings S, Wareham NJ (2007) Incidence of type 2 diabetes in England and its association with baseline impaired fasting glucose: the Ely study 1990-2000. Diabet Med 24:200-207

28. Valdes S, Botas P, Delgado E, Alvarez F, Cadorniga FD (2007) Population-based incidence of type 2 diabetes in Northern Spain: the Asturias Study. Diabetes Care 30:2258-2263

29. Soderberg S, Zimmet P, Tuomilehto J et al (2004) High incidence of type 2 diabetes and increasing conversion rates from impaired fasting glucose and impaired glucose tolerance to diabetes in Mauritius. J Intern Med 256:37-47

30. Meigs JB, Muller DC, Nathan DM, Blake DR, Andres R (2003) The natural history of progression from normal glucose tolerance to type 2 diabetes in the Baltimore Longitudinal Study of Aging. Baltimore Longitudinal Study of Aging Diabetes 52:1475-1484

31. Qiao Q, Lindstrom J, Valle TT, Tuomilehto J (2003) Progression to clinically diagnosed and treated diabetes from impaired glucose tolerance and impaired fasting glycaemia. Diabet Med 20:10271033

32. Abdul-Ghani MA, Jenkinson CP, Richardson DK, Tripathy D, DeFronzo RA (2006) Insulin secretion and action in subjects with impaired fasting glucose and impaired glucose tolerance: results from the Veterans Administration Genetic Epidemiology Study. Diabetes 55:1430-1435

33. Hu G, Lindstrom J, Valle TT et al (2004) Physical activity, body mass index, and risk of type 2 diabetes in patients with normal or impaired glucose regulation. Arch Intern Med 164:892-896

34. Healy GN, Dunstan DW, Shaw JE, Zimmet PZ, Owen N (2006) Beneficial associations of physical activity with 2-h but not fasting blood glucose in Australian adults. Diabetes Care 29: 2598-2604

35. Mishra GD, Carrigan G, Brown WJ, Barnett AG, Dobson AJ (2007) Short-term weight change and the incidence of diabetes in midlife: results from the Australian Longitudinal Study on Women's Health. Diabetes Care 30:1418-1424

36. Hamman RF, Wing RR, Edelstein SL et al (2006) Effect of weight loss with lifestyle intervention on risk of diabetes. Diabetes Care 29:2102-2107

37. Wannamethee SG, Shaper AG (1999) Weight change and duration of overweight and obesity in the incidence of type 2 diabetes. Diabetes Care 22:1266-1272

38. Tirosh A, Rudich A, Shochat $T$ et al (2007) Changes in triglyceride levels and risk for coronary heart disease in young men. Ann Intern Med 147:377-385 